\title{
A Classification and Performance Comparison of Mobility Models for Ad Hoc Networks"
}

\author{
Emre Atsan and Öznur Özkasap \\ Koç University, Department of Computer Engineering \\ Istanbul, Turkey \\ \{eatsan, oozkasap\}@ku.edu.tr
}

\begin{abstract}
In mobile ad hoc network research, simulation plays an important role in determining the network characteristics and measuring performance. On the other hand, unrealistic simulation conditions may be misleading, instead of being explanatory. For this reason, constructing simulation models closer to the real circumstances is very significant. Movement behavior of mobile entities is one of the most important concepts for the realistic simulation scenarios in mobile ad hoc networks. In this study, we first provide a survey and a new hybrid classification of existing mobility models in the literature. We implemented the random direction and boundless simulation area models on Scalable Wireless Ad Hoc Network Simulator (SWANS) and conducted simulations of Ad Hoc On-Demand Distance Vector (AODV) protocol for these as well as the random walk and random waypoint models. Our comparative results for the mobility models are discussed on a variety of simulation settings and parameters.
\end{abstract}

\section{Introduction}

Wireless and mobile ad hoc networks turn out to be the first option for a wide range of application areas, such as military, environmental, health, home automation and security [1]. Everyday, wireless devices, such as Bluetooth[2]-enabled cell phones, PDAs, and laptops become more popular and indispensable. Wireless Local Area Networks are a necessity even for home users due to the increase in the number of personal wireless electronic devices. In some cases, it turns out to be impossible or too costly to deploy permanent infrastructures (i.e. wireless routers, satellite links, GSM [3] networks) for a wireless network. For several military and civil applications, networking the mobile or static nodes with wireless links in an ad hoc manner can be necessary and/or effective [4].

The idea behind networking wireless nodes in an ad hoc manner dates back to the DARPA packet radio network research [5]. From those days to nowadays, there had been numerous research on mobile ad hoc networks (MANETs) including working groups [6]. Researchers use simulation tools $[7,8,9,10]$ to certify their algorithms and evaluate their performances. These simulation tools offer models that illustrate the

* This work is supported in part by TUBITAK (The Scientific and Technical Research Council of Turkey) under CAREER Award Grant 104E064. 
characteristics of a mobile ad hoc network. Besides network protocol details, communication channel properties, and spatial properties of simulation area, mobility modeling of nodes plays an important role in the characteristic of a MANET simulation. As argued in $[13,14,15]$, choice of a mobility model may affect the results of simulations significantly. Therefore, a better understanding of the behavior of mobility models and using the appropriate ones give us a chance to achieve realistic conclusions from simulations, which improves the validity of the results.

Several mobility models have been proposed for mobile ad hoc network simulations $[11,14,15,16,18,19,24]$ and various surveys $[13,16]$ have been published about them. In these surveys, authors have offered different classifications of the mobility models by pointing out several aspects. In [13], Camp et.al classified the models based on their generation methods (traces and synthetic models) and social behaviors (entity and group models). Bettstetter [16] derived a concept map for the classification of mobility models. In addition, [16] argues a classification model depending on the degree of randomness. Zheng et.al [17] use the same classification criteria founded upon randomness level. Lastly, a recent work [18] makes another classification for mobility models.

In this study, firstly we provide a survey and a new hybrid classification of mobility models. We discuss single entity (i.e. a node's movement is independent of other nodes) mobility models with their behavioral analysis studied recently [20,21], and show the resulting node traces for various simulation settings. We implemented the random direction and boundless simulation area models on SWANS (Scalable Wireless Ad Hoc Network Simulator) and conducted simulations of Ad Hoc On-Demand Distance Vector (AODV) protocol for these as well as the random walk and random waypoint models. Our comparative results for the mobility models are discussed on a variety of simulation settings and parameters. The remainder of this paper is organized as follows. Section 2 includes our classification of mobility models, and review of existing single entity mobility models. In Section 3, we present our simulation results of AODV using different mobility models. We aim to convey the importance of choosing the appropriate mobility model to obtain useful conclusions from ad hoc simulations. Section 4 concludes the paper.

\section{Mobility Models}

There exists different classification criteria proposed by several studies $[13,16,17,18]$ for mobility models in the literature. Table 1 shows a summary of these criteria. We bring these together to form a hybrid classification as depicted in Fig. 1.

\subsection{Traces}

Traces are pre-determined mobility patterns that are observed in real life. For ad hoc networks, tracing the actual behavior of mobile nodes is a hard process and researchers mostly use synthetic models [16]. Traces hardly let researchers to change simulation parameters, which can be a disadvantage for performance analysis of ad hoc networks. 
Table 1. Different criteria proposed for mobility model classifications

\begin{tabular}{ll}
\hline Classification Criteria & Classifications \\
\hline Generation of Mobility & Traces \\
& Synthetic Models \\
\hline Social Behavior of & Group Models \\
Mobile Nodes & Entity Models \\
\hline Degree of Randomness & Total pseudo-random movement process (Statistical Models) \\
& Bounded pseudo-random process \\
& (Constrained Topology based Models) \\
& Trace-based movement Model \\
\hline General & Integrated Mobility Models \\
\hline
\end{tabular}

\subsection{Synthetic Mobility Models}

a. Random Walk Mobility Model: The mathematical principles of the Random Walk Mobility Model go back to the Einstein's works on Brownian motion [13]. In some sources, this mobility model is called as Brownian Motion Mobility Model [19] or Brownian Walk [18]. In this mobility model, entities (mobile nodes) move randomly choosing a speed and direction from pre-defined ranges ([minspeed, maxspeed] and $[0,2 \pi]$, respectively) in constant time intervals $(\Delta t)$. Border behavior of this model is defined as bounce-back [13]. This means that when a mobile node reaches to a simulation boundary, it bounces back to simulation area. We place Random Walk Mobility model into the intersection of statistical and entity concepts in our classification chart (Fig. 1).

Due to its simplicity of implementation, Random Walk is a widely used mobility model in simulations. On the other hand, because of its memoryless behavior (i.e. decision of the next state doesn't depend on previous states) it creates unrealistic mobility patterns with sharp turns and sudden stops. It also exhibits a static behavior when it has a lower velocity range with low continuation. In graphs of Fig.2, it is

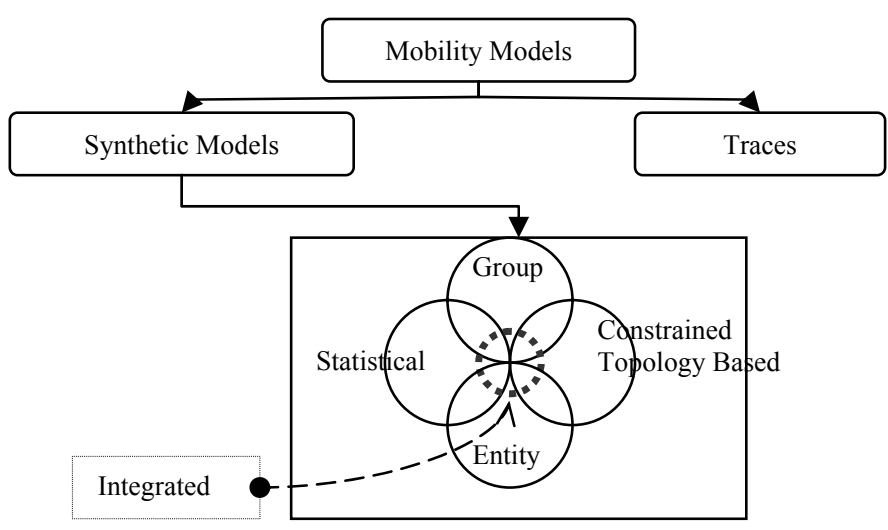

Fig. 1. Hybrid classification structure 
shown that lower velocity ranges cause a static behavior. However, increasing the speed range to higher values provides a better spatial distribution. This kind of behavior may lead to an unconnected network if the simulation area is large and number of nodes is small (Fig. 2).

b. Random Waypoint Mobility Model: Random Waypoint Mobility is the most widely used model in simulations by the research community [13]. In this model, a mobile node selects a random position $(x, y)$ in the simulation area as a destination point and a velocity $(v)$ from a uniformly distributed range [speedmin, speedmax]. Then node starts to travel to the chosen destination point with the constant selected speed, $v$. When the node arrives to the destination point, it pauses for a specific time (pause_time) defined as a simulation parameter. After this time, node selects a new destination and speed and repeats the process [26]. Random Waypoint Mobility Model is placed into the intersection of Entity and Statistical models in our classification.

Studies on the properties of Random Waypoint Model show that it creates a non-homogenous spatial distribution of nodes if it is used with specific (e.g. delete and bounce-back)
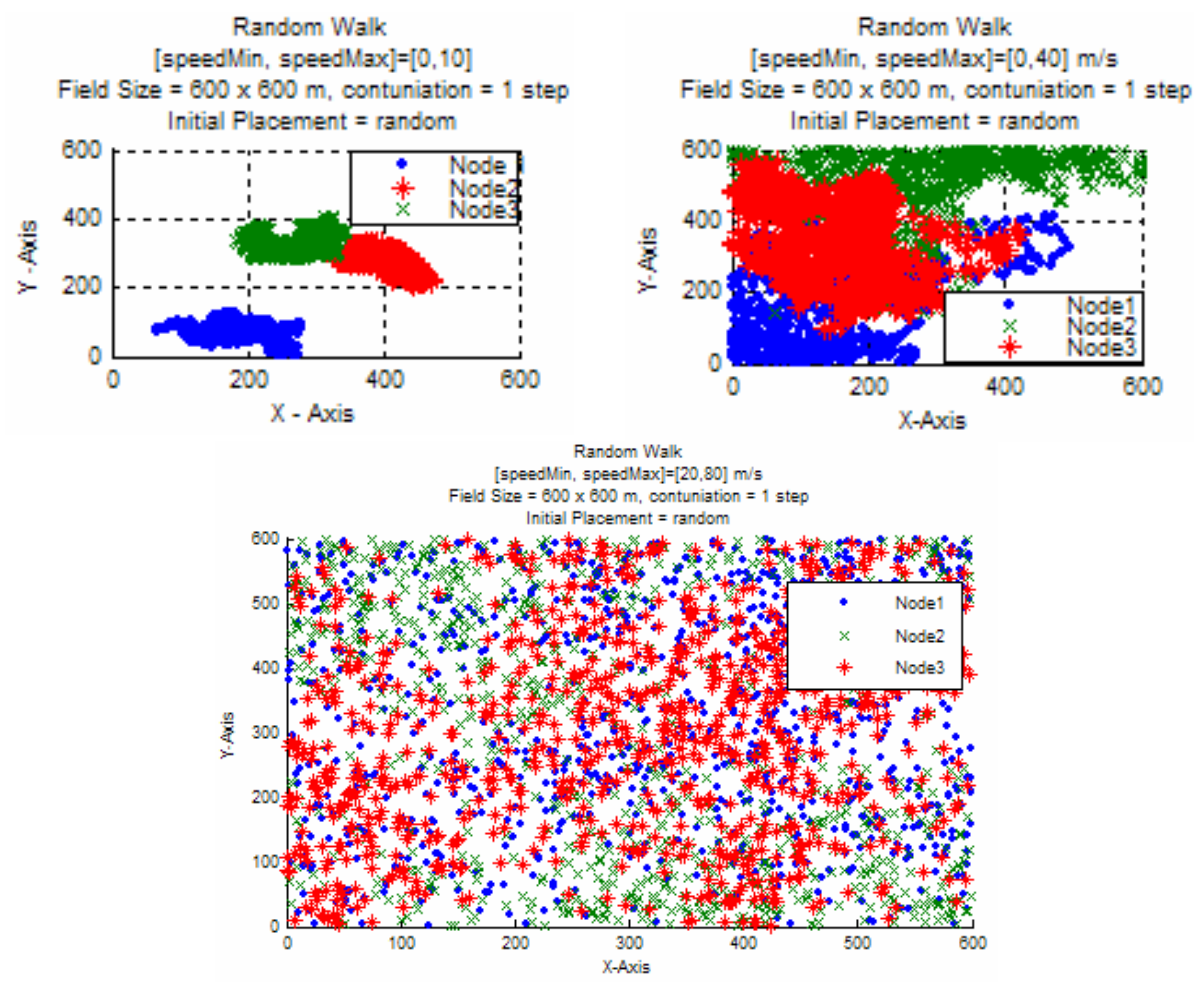

Fig. 2. Traces of Random Walk with speed ranges $[0,10],[0,40]$ and $[20,80] \mathrm{m} / \mathrm{s}$ in a field size of $600 \mathrm{~m} \times 600 \mathrm{~m}$. Simulations performed using JIST/SWANS tool's [8] implementation of Random Walk Mobility Model. Continuation value shows the speed and direction change frequency. Continuation = 1 means "change speed and direction in 1 second". 
border behavior selections [20]. This means that nodes tend to concentrate on the middle of the field. To avoid this problem, it is suggested to use wrap-around border behavior [16]. Wrap-around border behavior creates a torus-like simulation area by wrapping the edges of area around [24]. Another statistical behavior of Random Waypoint Mobility Model is described in [14]. During their simulations of AODV, Royer et al. discovered a strange characteristic of this model. The average number of neighbors recognized at a given node periodically increases and decreases during the simulation. Frequency of this increase-decrease is relative to the speed of the nodes. This behavior creates a situation in which nodes unite and separate in the center of the simulation area. Authors called this situation density waves.

Fig. 3 shows our simulation traces of Random Waypoint model in JIST/SWANS [8] simulation tool. Bounce-back border behavior was implemented in these simulations. Charts were formed by 14000 and 65000 data points, respectively. Each simulation ran for 500 simulation seconds. Our results confirm the previously discussed [20] behaviors of Random Waypoint mobility model. In Fig. 3, it can be observed that nodes generally tend to concentrate in the middle of the simulation area. Furthermore, when the speed range and hence the average speed of mobile nodes is increased from $[0,20]$ to $[40,80]$, nodes' distribution in the center of the area becomes denser.

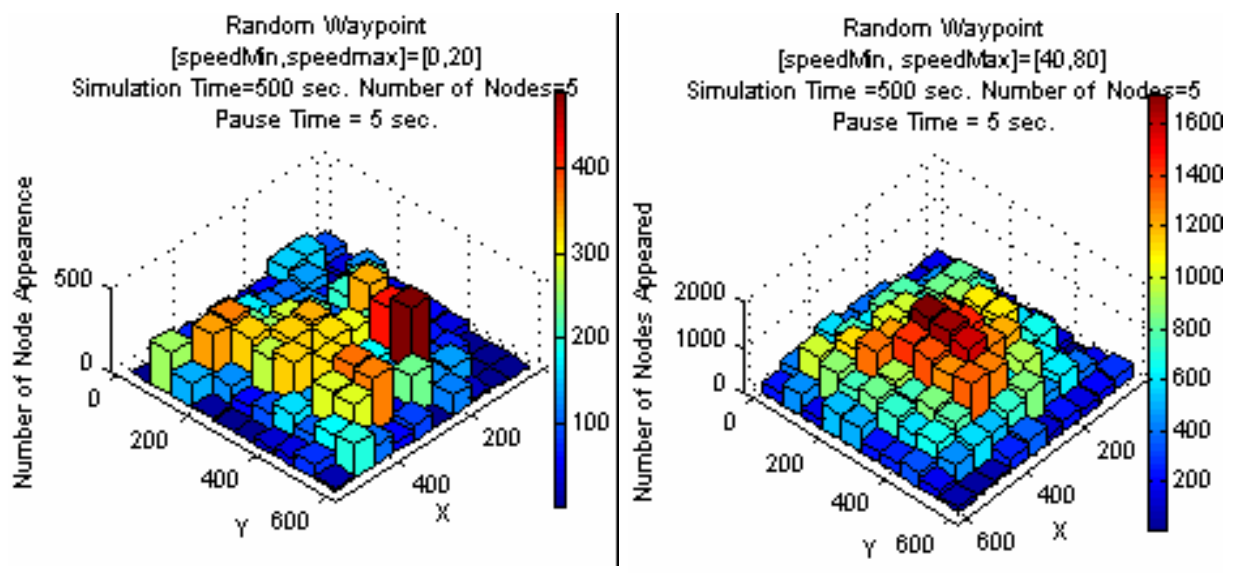

Fig. 3. Density distributions of 5 nodes in 600 x 600 simulation field (Random Waypoint Mobility Model). Speed ranges are [0,40] and [40, 80] $\mathrm{m} / \mathrm{sec}$, respectively. Pause time is 5 simulation seconds.

c. Random Direction Mobility Model: Random Direction Mobility Model was proposed in [14]. It is designed to overcome the density waves problem faced in Random Waypoint model. In this mobility model, a node selects a random direction uniformly between $[0,2 \pi]$. Then it chooses a velocity between [speedmin, speed$\max ]$, again uniformly. Node continues to travel until it reaches the boundary of the simulation area. Once it reaches the boundary, it rests for a given pause time and selects a new direction between $[0, \pi]$. The direction is limited to $\pi$ because the node is already on the boundary and cannot exit from the simulation area. We place Random Direction Mobility model into the intersection of statistical and entity models in our classification. 


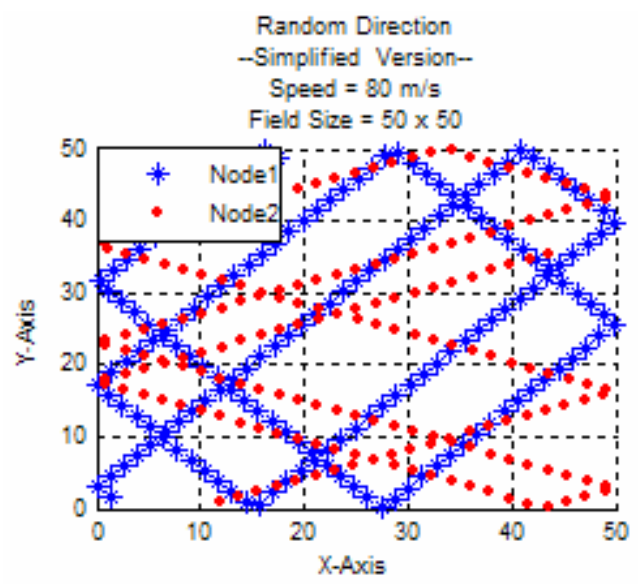

Fig. 4. Movement trace of two nodes. Simplified version of Random Walk Mobility Model used in $50 \times 50$ simulation area.

There exist several variations of Random Direction Model in the literature. "Modified Random Direction Mobility Model" proposed in [14] doesn't force the mobile node to travel to the boundary for changing speed and direction. A node can select its new direction and speed anywhere on its path. A simplified version of Random Direction model was used by Haas and Pearlman in [12]. In this version, all the nodes have a common constant speed $v$ and move with an initial direction $(\varphi)$ selected from a uniformly distributed range $[0,2 \pi]$. When a node reaches the boundary, it reflects back into the simulation area with an angle $(-\varphi)$, if it is on a vertical edge; $(\pi-\varphi)$, if it is on a horizontal edge. A simulation trace of simplified version for two nodes is shown in Fig. 4.

d. Boundless Simulation Area Mobility Model: In Boundless Simulation Area Mobility Model, different from Random Direction, Waypoint and Walk, there exists a relationship between the previous state of the node and the current state [24]. In other words, the new direction and speed values are dependent on the previous direction and speed values of the node, respectively. In every $\Delta t$ time period, new values of the direction and speed are assigned according to the given formulas below:

$$
\begin{gathered}
v(\mathrm{t}+\Delta \mathrm{t})=\min \left[\max (v(\mathrm{t})+\Delta v, 0), \mathrm{V}_{\max }\right] \\
\varphi(\mathrm{t}+\Delta \mathrm{t})=\varphi(\mathrm{t})+\Delta \varphi \\
\mathrm{x}(\mathrm{t}+\Delta \mathrm{t})=\mathrm{x}(\mathrm{t})+\mathrm{v}(\mathrm{t}) * \cos [\varphi(\mathrm{t})] \\
\mathrm{y}(\mathrm{t}+\Delta \mathrm{t})=\mathrm{y}(\mathrm{t})+\mathrm{v}(\mathrm{t}) * \sin [\varphi(\mathrm{t})]
\end{gathered}
$$

$\mathrm{V}_{\text {max }}$ : maximum defined velocity of the simulation.

$\Delta v$ : change in the velocity of the node in $\Delta \mathrm{t}$ time period. It is selected from $\left[-\mathrm{a}_{\mathrm{MAX}} * \Delta \mathrm{t}\right.$, $\left.\mathrm{a}_{\mathrm{MAX}} \Delta \mathrm{t}\right]$ range uniformly, where $\mathrm{a}_{\mathrm{MAX}}$ is the maximum defined acceleration (per $\Delta \mathrm{t}^{2}$ ) parameter for this simulation. 
$\Delta \varphi$ : change in the direction of the node in $\Delta \mathrm{t}$ time period. It is selected from the range $\left[-\omega_{\mathrm{MAX}} * \Delta t, \omega_{\mathrm{MAX}} \Delta \mathrm{t}\right]$ uniformly, where $\omega_{\mathrm{MAX}}$ is the maximum allowed angular change in the direction of mobile node in $\Delta t$ time period [13].

Another important property of this mobility model is its border behavior. When a node reaches to a boundary of the simulation area, it doesn't bounce back into simulation area; instead it continues to travel and reappears on the other side of the simulation area. This is similar to a torus-like simulation area as illustrated in Fig.5.
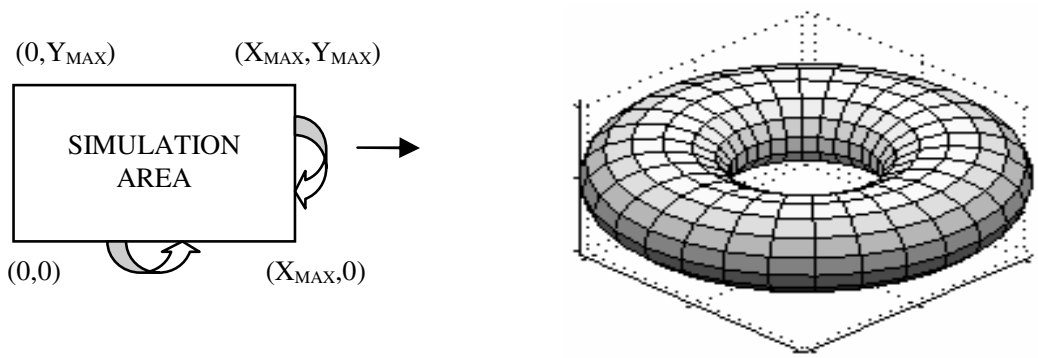

Fig. 5. Construction of a torus-like Boundless Simulation Area from a 2D simulation area

Boundless Simulation Area Mobility Model creates more realistic node movement traces than Random Walk, Waypoint models, because it depends on the previous velocity and direction of the node. Sharp turns and sudden stops can be eliminated in Boundless Simulation Area model by using this state-dependency of the nodes. We place Boundless Simulation Area Mobility Model into the intersection of statistical and entity models in our classification.

In Fig. 6, our simulation results depicting the movement behavior of a node for Boundless Simulation Area Mobility Model are given. Long lines cutting the simulation

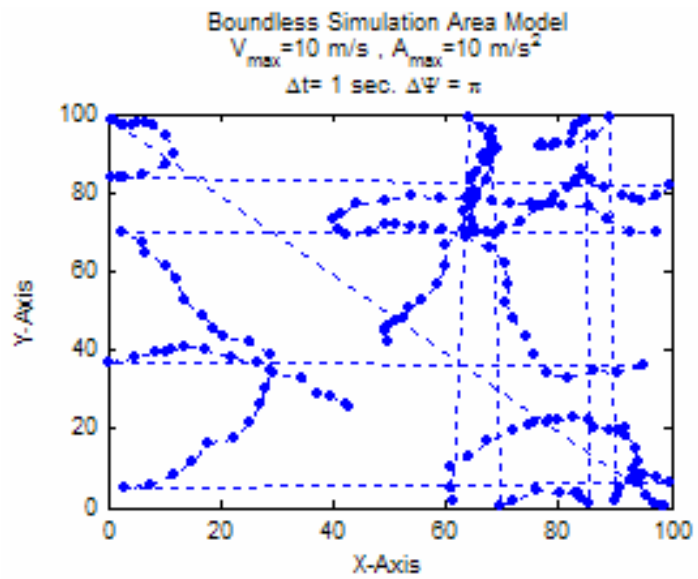

Fig. 6. Simulation trace of Boundless Simulation Area Mobility Model 
area from one side to another indicates the reappearance of a node on the other side of the simulation area.

e. City Section Mobility Model: City section mobility model was first proposed by Davies [11]. It uses a simulation area that represents street network of a city. A node is not allowed to choose any point on the graph. It should select a point that is on some street network of the city. After selecting this destination point, the travel path is determined by an algorithm, which calculates the shortest travel time between source and destination points. After reaching to the destination point, node waits there for a defined pause time and randomly chooses another destination on the street network and repeats the process. In addition to this behavior, in City Section Mobility model, a mobile node should obey some pre-defined driving characteristics, such as "speed limit" and "minimum distance allowed between any two nodes". These rules and the use of pre-defined paths (street network) make the movement behavior of the mobile node similar to a vehicle movement in a city central [11]. We place City Section Mobility Model into the intersection of constrained topology based and entity models in our classification.

f. Smooth Random Mobility Model: Smooth Random Mobility Model was originally proposed by Bettstetter [16]. The idea behind the design of this mobility model is to "make the movement of users (pedestrians and vehicles) more smooth and realistic than previously known random models." [16]. Two stochastic processes are used for direction and speed control in which the new values of speed and direction are correlated with the previous ones. This correlation is what makes the movement smoother, because it omits the unrealistic sharp turns and sudden stops in simple random movement models [16]. Another important point that Bettstetter added to his mobility model is the preferred speeds concept. Users select their new target speed from a range $\left[0, V_{\text {max }}\right]$ uniformly, except $V_{\text {pref }}$ has a higher probability than other speed values. By this way, some typical speed patterns can be created, which resembles the movement behaviors of the vehicles and pedestrians. Bettstetter extends his model by adding two new concepts in it. He assumes that in reality generally speed and direction change are not independent from each other. For this purpose, he offers a correlation between speed change and direction change: "Stop-turn-and-go" behavior and "slowdown of turning nodes" notions. Stop-turn-and-go behavior describes a mathematical model of the reality: a stop of a vehicle often followed by a direction change. So whenever a node comes to a stop $(v=0)$, then node choose a new target direction to move. The selection of the node's new direction is decided by the direction behavior model defined in the same paper [16]. Slowdown of the turning nodes concept was inspired by the physics laws. This means that if a vehicle stops at a given time and point, than it should first slowdown in a given period of time. This concept gives the Smooth Random Mobility Model a more realistic movement behavior than Random Walk and Waypoint models.

Smooth Random Mobility model can be summarized as a (statistical) random mobility model with better movement dynamics of the nodes. It was proposed as a cooperation of simple random mobility models and realistic and complicated mobility models of transportation research [16]. We place Smooth Random Mobility Model into the intersection of statistical and entity models in our classification. 
g. Obstacle Mobility Model: Obstacle Mobility Model [15] is designed to model the mobility patterns that may resemble the real world paths on real world topographies. Model provides obstacles inside the simulation area. These obstacles both restrict the movements of the mobile nodes and the wireless transmission range. Nodes are supposed to follow the given provided pathways; instead of the random trajectories. In this model, the simulation area and the pathways are constructed by using the Voronoi diagram of the given obstacles. Obstacle and pathway construction using Voronoi diagrams are described in [15]. Model uses a reachability matrix to represent the transmission possibility between pairs of nodes. Obstacles are assumed opaque and totally block the signal propagation. After defining the pathways from obstacles (using Voronoi graphs) and calculating reachability matrix, each time a node transmits a packet; the model determines whether the intended recipient of the packet is within the reachability matrix of the transmitting node. If so, reception of packet is completed. We place Obstacle Mobility model into the intersection of constrained topology based and entity models in our classification.

\section{Simulation Study and Analysis Results}

Our simulations have been performed using the SWANS Network Simulator [8]. SWANS is built on Java-based simulation framework JIST (Java in Simulation Time). We investigate the effects of different mobility models on ad hoc network performance using AODV [22] routing protocol as the route establishment choice. We use the available AODV implementation in SWANS, details of which can be found in [25].

For our comparisons of AODV performance with different mobility models, we used Random Walk, Random Waypoint, Random Direction and Boundless Simulation Area Mobility models. First two models are already available in SWANS, and we contributed Random Direction and Boundless Simulation Area models to the simulator. For Random Direction Mobility Model, we implemented the simplified version (constant velocity and reflection) used by Haas and Perlman in [12]. For Boundless Simulation Model, we used the Haas' proposal [24] as a reference for our implementation. Parameters of our simulation settings are listed in Table 2.

In Figures 7 to 9, we present our simulation results. For Figures 7, 8.a and 9.a, the size of the simulation field $(1000 \mathrm{~m} \times 1000 \mathrm{~m})$, pause times of the mobility models, minimum and maximum velocity ranges are same and constant. For Figures 8.b and 9.b,

Table 2. Simulation Default values in SWANS

\begin{tabular}{ll|ll}
\hline Routing Protocol & AODV & Radio Trans. Strength & $15 \mathrm{dBm}$ \\
Transport Protocol & UDP [23] & Radio Reception Sensitivity & $-91 \mathrm{dBm}$ \\
Fading & None & Radio Reception Threshold & $-81 \mathrm{dBm}$ \\
Path-loss & Freespace & Packet Loss & None \\
Radio Frequency & $2.4 \mathrm{GHz}$ & Placement (Node Distribution) & Random \\
Radio Trans. Bandwidth & $1 \mathrm{Mb} / \mathrm{s}$ & $\begin{array}{l}\text { Packet send rate per minute for } \\
\text { node }\end{array}$ & 1.0 \\
\hline
\end{tabular}


Table 3. Simulation variables that are kept constant throughout the AODV simulations

\begin{tabular}{|c|c|c|c|}
\hline Pause time (for related models) & $10 \mathrm{sec}$. & $\begin{array}{l}\text { Maximum acceleration } \\
\text { (for Boundless } S . \text { ) }\end{array}$ & $10 \mathrm{~m} / \mathrm{s}^{2}$ \\
\hline [speedmin, speedmax] & $\begin{array}{l}{[20,80]} \\
\mathrm{m} / \mathrm{s}\end{array}$ & $\begin{array}{l}\text { Max. allowed angular change } \\
\text { (for Boundless } S \text {.) }\end{array}$ & $\Pi / 2$ \\
\hline
\end{tabular}

number of nodes in the simulation area is constant (50 nodes) and randomly distributed during the initialization. Some of the simulation variables that are constant in all of the simulations are given in Table 3. In these simulations, we run 10 different simulations for every mobility model and calculated the average of their results.

\subsection{Discussion of Simulation Results}

Fig.7 illustrates that increasing the number of nodes in the constant sized simulation area causes dissimilar increase in the message activity per node for each mobility model. For the whole simulation, Random Direction mobility model creates a higher message activity. On the other hand, Boundless Simulation Area creates the lowest. We know that message activity is dominated by the sent RREQ messages for AODV implementation which is also discussed in [25]. At this point we investigate the big difference in message activity for different mobility models.

First of all, this difference can result from the border characteristic of the Boundless Simulation Area model. For example, if two nodes are at the two end of the simulation area, they are not within each other's transmission range in Random Direction, Walk and Waypoint models. On the other hand, because of the wrapping of simulation borders,

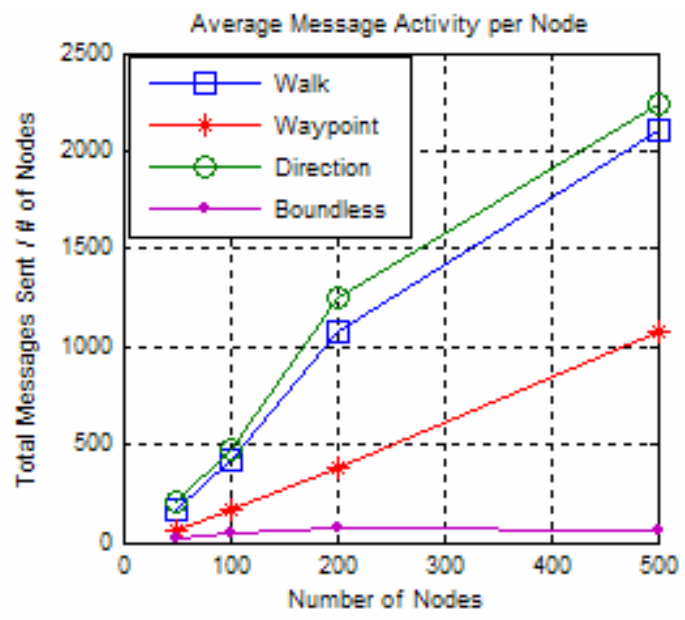

Fig. 7. Average message sent per node for different values of number of nodes in the simulation (all other variables held constant). "Total Messages Sent" is the number of all control messages (RREQ, RREP, RERR, and HELLO) sent by the nodes in AODV algorithm. Data packets are not included in the "Total Messages Sent". 
these two nodes became neighbors in Boundless Simulation Area. So, for the same size of the simulation area, nodes near the boundary have two times line-of-sight if they are moving based on the Boundless Simulation Area mobility model. If the line-of-sight (assume that it is equivalent to transmission range in this special case) of the nodes at the boundary increases, than the number of RREQ messages sent decreases. This is because if the destination point is far or not accessible, then sender node starts to transmit large number of RREQ messages in AODV routing. Increasing the line-of-sight for nodes automatically decreases the probability of inaccessible destination nodes.
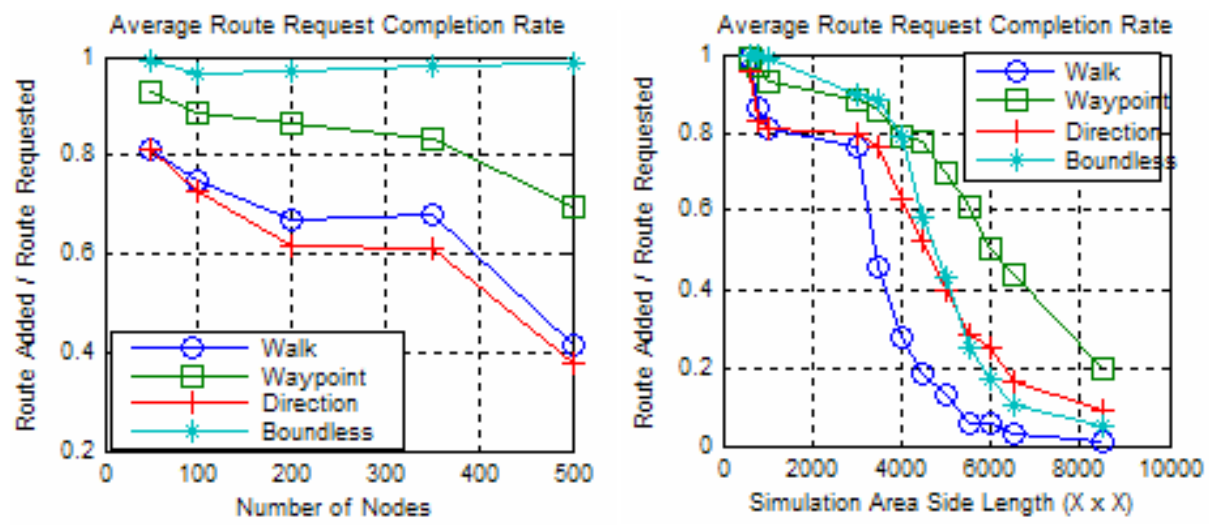

Fig. 8. Average Route Request Completion Rate (for varying number of nodes with constant field size) \& (for varying simulation area sizes with constant number of mobile nodes in simulations) gives the rate of routes added (found) to requested routes

In Fig.8, we present a new metric for the performance of AODV simulations under different mobility models. Average Route Request Completion Rate is the ratio of completed routes to the all requested routes. This is an important performance criterion for the route finding performance of AODV protocol. As we can easily observe, for small number of node sizes, each mobility model gives good completion rates for requested routes. However, for larger node sizes, Random Direction and Walk mobility models starts to give a lot of no-path found errors. As discussed before, Boundless Simulation Area model behaves so accurate for increasing number of nodes. This may be because of the same reason discussed before: increased line-of-sight at the borders automatically decreases the inaccessible node quantity. For this reason, Boundless Simulation area mobility model has a nearly perfect route request completion ratio for small simulation area sizes. On the other hand, when we increase the simulation area size, while keeping number of nodes constant, we observe that Boundless Simulation Area model starts to loose its "wrapped area" and "greater line-of-sight" advantage. This means that for very large simulation area, having a connection with the nodes on the other boundary of the area doesn't have a big impact on the inaccessible node number. This is because, when the area grows, the probability of nodes' being near the boundary decreases. Therefore, after some very large field sizes, Boundless Simulation Area is expected to behave differently. As we can easily observe for small sizes of simulation area, all of the mobility models give good (nearly perfect) completion 
ratios. This is expected because of the high node density in the simulation area for small area sizes.

Fig. 8 indicates that Random Waypoint model behaves much better than other two random models (random direction and random walk) for varying number of simulation area sizes. This is because of the interesting spatial behavior of the Random Waypoint mobility model, as discussed in Section 2 and originally in [20]. Because of this mathematically proved, behavior of Random Waypoint mobility model, nodes tend to concentrate on the middle of the simulation area, which causes a decrease in the average number of inaccessible nodes for random waypoint model. As a result, Random Waypoint mobility model has a tidier behavior for increasing simulation size, which shows us that Random Waypoint model can be better solution for the simulations that assumes the simulation area size does not a factor for resulting performance values.
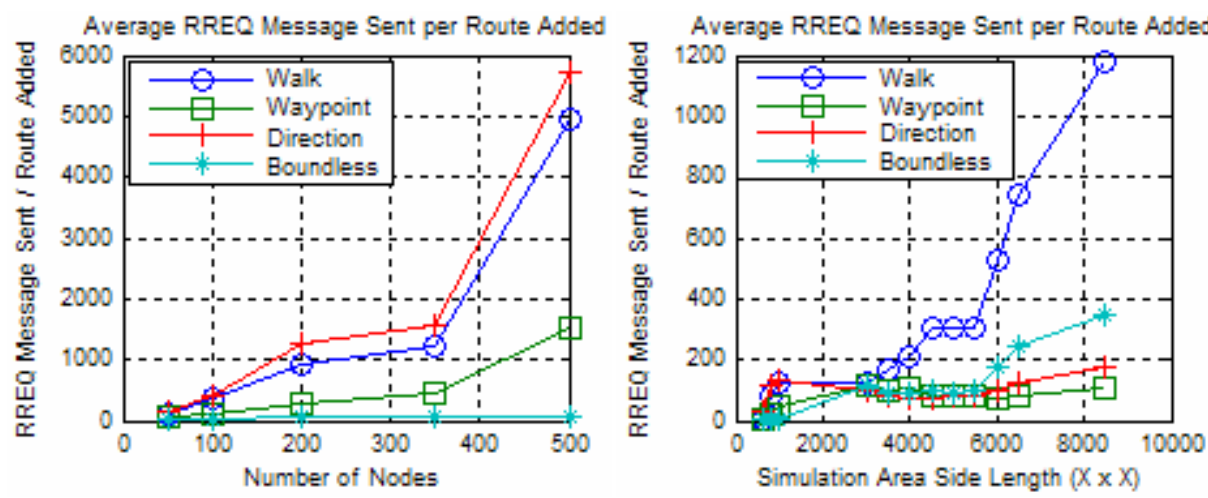

Fig. 9. Average RREQ message sent per route found (for varying number of nodes with constant field size) \& (for varying simulation area sizes with constant number of mobile nodes in simulations) is a performance metric that indicates the redundant message creation on the network for a route added. RREQ message is a control message used in AODV routing protocol. Basically, it is sent from the initiator of the route request to its neighbors. Then every neighbor continues this process until the destination is found.

In Fig.9, we present another new metric for the performance of AODV routing: Average RREQ Message Sent per Route Added. RREQ is a control message used in AODV. Basically, it is sent from the initiator of the route request to its neighbors. Then every neighbor continues this process until the destination found. If a source node cannot get a RREP message for a given period of time, it retransmits the RREQ message to the network. Average RREQ message sent per route found (or added) is a measure of the efficient use of network resources for finding a route to the destination node. If too much RREQ message is sent to the network to find a route to the destination, it would not be a very efficient route finding. So, this parameter is an important performance concept for AODV routing protocol which leads to different performance results for different mobility models. From Fig.9, it can be easily observed that Boundless Simulation Area model gives nearly perfect simulation performance for increasing number of nodes. Random Waypoint model has an average performance 
with high stability in this category. On the other hand, Random Walk and Random Direction mobility models give poor efficiency ratios for large node sizes.

While increasing field size and keeping number of nodes constant in the simulation area, Random Waypoint and Boundless Simulation Area mobility models give efficient performance values for AODV, in contrast to the Random Direction and Random Walk models. However, for very large field sizes, the efficiency of the nodes using Boundless Simulation Area model starts to fall significantly. This fall in the efficiency of AODV routing is again caused by losing "wrapped area" and "greater line-of-sight" advantage of this model for very large field sizes as discussed before. On the other hand, Random Waypoint Model keeps its efficiency even for $8500 \mathrm{~m} \mathrm{x}$ $8500 \mathrm{~m}$ field. This is mainly due to the nodes' tendency to concentrate on the center of simulation area. This spatial distribution characteristic of nodes was discussed in [20].

These results show us that selection of the mobility model for simulations of ad hoc networks can hugely affect the results. For example, if a researcher wants to use AODV routing protocol for his wireless sensor network structure that nodes move similar to the Brownian motion of molecular particles, for large number of nodes, AODV can lead to poor network resource efficiencies in real life (based on the Random Walk mobility model results in Fig.9). However, this doesn't mean that AODV is an inefficient routing protocol for all cases. The important thing here is to find the most realistic mobility model for the desired case.

\section{Conclusions}

Everyday mobile ad hoc networking becomes more and more popular and extends its range of application areas. As a result of this, understanding and realistically simulating the behavior of mobile networks is very important for the researchers in the progress of designing better algorithms for ad hoc networks. Realistic simulations need realistic mobility models and better simulation tools. Numerous advantages of using simulation tools for performance analysis of networks [15] put the mobility modeling and its statistical analysis into an important level for the future.

In this paper, we first provide a survey and a new hybrid classification of existing mobility models in the literature. It has been also shown that when the mobility behaviors of nodes change in an ad hoc network, the performance of the network can be vastly affected from this. So that choosing a realistic mobility model for network simulations plays an important role on the validity of the simulation results. For an ad hoc network that propagates data based on AODV routing protocol, when different mobility models applied, so different performance results appear with varying number of nodes or simulation area size. Based on our simulations, we propose that for all of the performance metrics simulated, Random Waypoint Mobility model has not the best performance; however it is the most consistent one for varying simulation values. Using Boundless Simulation Area Mobility Model, for some performance metrics AODV routing protocol performed remarkably well. But, varying factors in the simulation environment enormously affected its performance values in simulations. Two other mobility models, Random Direction and Random Walk, showed the worst performances for AODV routing in simulations. We also demonstrated that Random Walk Mobility is the most affected model from the increase in the simulation size for the performance metrics investigated in this study. 


\section{References}

1. I. F. Akyildiz, W. Su, Y. Sankarasubramaniam, E. Cayirci. Wireless sensor networks: a survey. Computer Networks, Vol. 38, pp. 393 - 422, March 2002.

2. The Official Bluetooth Wireless Info website. http://www.bluetooth.com/.

3. Global System for Mobile Communications (GSM) official website. http://www. Gsm world. com/.

4. M. Frodigh, P. Johansson, P. Larsson. Wireless ad hoc networking-The art of networking without a network. Ericsson Review No. 4, 2000.

5. J. Jubin, J. D. Tornow. The DARPA Packet Radio Network Protocols. In Proceedings of the IEEE, Vol. 75, 1, pp. 21-32, January, 1987.

6. IETF MANET Group. http://www.ietf.org/html.charters/manet-charter.html.

7. QualNet simulation environment. http://www.scalable-networks.com

8. JIST/ SWANS (Java in Simulation Time / Scalable Wireless Ad hoc Network Simulator) simulation environment. Available at http://jist.ece.cornell.edu/.

9. The Network Simulator - ns-2. Available at http://www.isi.edu/nsnam/ns/.

10. L. Bajaj, M. Takai, R. Ahuja, K. Tang, R. Bagrodia, and M. Gerla. GlomoSim: A Scalable Network Simulation Environment. Technical Report, \#990027, UCLA, 1997.

11. V. Davies. Evaluating mobility models within an ad hoc network. Master's thesis, Colorado School of Mines, 2000.

12. Z. J. Haas and M. R. Pearlman. The performance of query control schemes for the Zone Routing Protocol. In Proceedings of ACM SIGCOMM, Vancouver, Canada, Sept. 1998.

13. T. Camp, J. Boleng, and V. Davies. A survey of mobility models for ad hoc network research. Wireless Comm. and Mobile Computing (WCMC), 2(5):483-502, 2002.

14. E. Royer, P.M. Melliar-Smith, and L. Moser. An analysis of the optimum node density for ad hoc mobile networks. In Proc.IEEE International Conference on Communications, 2001.

15. A. Jardosh, E. Royer, K. Almeroth, and S. Suri. Towards realistic mobility models for mobile ad hoc networks. In MobiCom'03, September 2003.

16. C. Bettstetter. Smooth is better than sharp: A random mobility model for simulation of wireless networks. In Proceedings of MSWiM'01. ACM, July 2001.

17. Q.Zheng, X. Hong, S. Ray. Recent Advances in Mobility Modeling for Mobile Ad Hoc Network Research. In ACMSE'04, April 2004.

18. I. Stepanov, P. J. Marron, K. Rothermal. Mobility Modeling of Outdoor Scenarios for MANETs. In Proceedings of ANSS'05, 2005.

19. M. Musolesi, S. Hailes, C. Mascolo. An Ad Hoc Mobility Model Founded on Social Network Theory. In MSWiM'04, October, 2004.

20. C. Bettstetter and C. Wagner, The Spatial Node Distribution of the Random Waypoint Model, Proceedings of the 1st German Workshop Mobile Ad Hoc Networks (WMAN), 2002.

21. C. Bettstetter, H. Hartenstein, and X. Perez-Costa, Stochastic Properties of the Random Waypoint Mobility Model, Wireless Networks 10, 555-567, 2004.

22. C. E. Perkins, E. M. Royer. The Ad Hoc On-Demand Distance Vector Protocol. In C.E. Perkins, editor, Ad hoc Networking, pp. 173-219. Addison-Wesley, 2000.

23. J. Postel. RFC 768: User Datagram Protocol (UDP). http://www.ietf.org/rfc/rfc768.txt.

24. Z. J. Haas. A new routing protocol for reconfigurable wireless networks. Proc.IEEE International Conference on Universal Personal Communications (ICUPC), Oct. 1997.

25. C. Lin. Documentation: AODV Routing Implementation for Scalable Wireless Ad-Hoc Network Simulation (SWANS). JIST/SWANS web site. http://jist.ece.cornell.edu/.

26. J. Broch, D. A. Maltz, D. Johnson, Y.-C. Hu, J. Jetcheva. A Performance Comparison of Multi-Hop Wireless Ad Hoc Network Routing Protocols. In Proceedings of the $4^{\text {th }}$ Annual ACM/IEEE International Conference on Mobile Computing and Networking (MobiCom), pages 85-97, Dallas, Texas, October 1998. 